\title{
A Comparative Analysis of the Educational Effectiveness of Leaflet and Website for Low-Literate Patients - A Case Study of Immigrant Mothers in Taipei
}

\author{
Yah-Ling Hung ${ }^{1}$, Kai-Ren Chen ${ }^{2}$, Catherine Stones ${ }^{3}$, and Thomas Cassidy ${ }^{3}$ \\ ${ }^{1}$ Dept. of Communication Arts, Fu Jen Catholic University, Taiwan \\ ${ }^{2}$ Dept. of Public Health, Fu Jen Catholic University, Taiwan \\ ${ }^{3}$ School of Design, University of Leeds, UK \\ $030872 @$ mail.fju.edu.tw
}

\begin{abstract}
Low health literacy has been associated with poor outcomes in health care. Recent research suggests that good health educational material can help to reduce the literacy barrier and enhance health outcome. Immigrant populations are vulnerable to serious health disparities, and language barriers may further exacerbate their limited health literacy in accessing health care information. Yet, ways to help low-literacy parents to look after their children by applying health educational material are still at an early stage of development. The purpose of this study is to compare the educational effectiveness of leaflet and website to deliver knowledge related to children allergy healthcare for immigrant mothers with low literacy, thus establishing design guidelines of health educational materials for low-literate patients. The study was implemented in five stages, including a focus group interview, the development of testing media, a reliability and validity test, a pre-post knowledge test, and a usability survey.The findings revealed the problems low-literate patients usually encountered at the clinic, the pediatric information they most needed, the way they were able to acquire knowledge of children's healthcare, and the media they usually used to access information. Moreover, the results demonstrated that the leaflet intervention, as well as the website intervention, had a positive outcome. However, there was no significant difference between the influence of design intervention made by the leaflet and by the website. The findings also showed that the criteria used by low-literate patients to evaluate health educational material could be analyzed in terms of the quality of information, presentation, and appeal. An assessment checklist related to the design of health educational materials for low-literate patients was also listed.
\end{abstract}

Keywords: Design Intervention, Health Literacy, Low-literate Health Education, Health Educational Material Design.

\section{Introduction}

\subsection{Research Background}

Low health literacy has been associated with poor outcomes in health care, which include higher health care costs, a worse assessment of one's own degree of health, 
and less success in managing chronic diseases (National Institute of Health, 2002). Recent research suggests that good health educational materials can help to reduce the literacy barrier and enhance health outcome, they can help modify attitudes, shape positive behaviors, and improve patients' self-prevention. However, the majority of health educational materials are constructed for well-educated users rather than those with low literacy skills. This begs the question of whether or not various health educational materials have the same effect on low-literate patients.

Immigrant populations are vulnerable to serious health disparities, and language barriers may further exacerbate their limited health literacy in accessing health care information. According to the Ministry of the Interior, a total of 44622 new immigrants were registered on the census of Taipei city in 2012, and their number of newborn babies was 14220 , which accounted for $5.87 \%$ of the total number of new babies born in Taipei. This research considers a specific group within Vietnamese and Chinese immigrant mothers in Taipei, most of who have low levels of education and have married into poor families. Therefore, their children might not get good care because of low socio-economic status. Recent surveys indicate that more than 20 per cent of children in Taiwan suffer from allergies. Children with chronic diseases are highly dependent on their parents in the management of their health care. Yet, ways to help low-literate parents to look after their children by applying robustly designed health educational materials are still at an early stage of development.

The past decade has seen a great proliferation of research into low-literacy health communication, such as information and communication technologies ( Mishra et al., 2007; Allison \& Ricardo, 2011 ), health education for low-literate patients (Andersen et al., 2008; Ndwe et al., 2010; Choi \& Bakken, 2010), consumer-centered health information design (Weitzman et al., 2009; Rubinelle et al., 2009), and servicelearning technology for low-literate patients (Houston et al., 2006; Wexler et al., 2011). Past studies related to low literacy health communication cover diverse disciplines ranging from health science to health education, information technology, and health care application. However, they mainly focus on exploring the effectiveness of health promotional platforms from the perspective of information providers and consumers, while comparative analyses of the educational effectiveness of various media for low-literate users from the perspective of designers are comparatively rare.

\subsection{Purpose of This Study}

The purpose of this study is to compare the educational effectiveness of leaflet and website to deliver knowledge related to children allergy healthcare for immigrant mothers with low-literacy, thus establishing design guidelines of health educational materials for low-literacy patients. Even though leaflet is relatively inexpensive to create and provides valuable information for populations, the benefit of its one-sizefits-all approach varies from person to person. With the recent advances in computer technology, website, which combine texts, pictures, audio, and video, seem to be welcome, but whether or not their versatile presentations affect low-literate patients' health knowledge is not yet known. Considering the issues of concern above, the primary research objectives of this study are described below: 
- To survey current strategies, methodologies, and tools to design health educational materials for low-literate patients

- To compare the educational effectiveness of leaflet and website to deliver children's health care knowledge to users with low literacy.

- To set up guidelines for the design of health educational materials for low-literate patients.

\section{$2 \quad$ Literature Review}

\subsection{Design Interventions (Leaflets VS. Websites)}

There has been a huge proliferation of research into health-based design interventions in the past decade, most of which focused on the educational effectiveness of paperbased and web-based media. For example, Jan et al. (2007) attempted to determine whether or not a web-based multimedia asthma educational and monitoring program would improve the knowledge and health status of children and caregivers. 164 pediatric patients with persistent asthma were enrolled for a 12-week controlled trial. The results showed that the adherence rates of therapeutic and diagnostic monitoring, the global assessment of asthma control, the knowledge of asthma self-management, and the quality of life of caregivers were all significantly higher in the intervention group. Moreover, Yardley et al. (2010) conducted two in-depth qualitative studies to compare the effectiveness of paper-media and web-based media for providing medical care for flu. 47 participants were randomly placed into two groups to explore the influence of the tested media that delivered health care information about cold or flu symptoms. The results showed that users often felt overwhelmed by the quantity of information provided in paper-based media, and they wanted to have greater control of how information is accessed in web-based media.

Since paper-based media are typically created for the general population, they do not really consider the specific characteristics of prospective consumers. On the contrary, web-based media is based upon the principle of "market segmentation" which aims to find a specific group of consumers for a particular product or service. Indeed, targeted information is well suited to meet the needs of targeted populations. Compared to the function of one-size-fits-all paper-based media, web-based media can tailor information which applies to people with similar characteristics, allowing interventions to effectively target high-risk groups. Furthermore, since displaying photographs and video clips in a web browser does not require the use of additional expensive hardware, it can be generated inexpensively. Conversely, printed educational booklets are costly to produce and update. (Richards et al. 1998; Ahern et al. 2010; Kreuter et al. 2010). 


\begin{tabular}{|l|l|l|}
\hline & Leaflet & Website \\
\hline 1 & Static Media & Dynamic media \\
\hline 2 & Portable & Portable but expensive (smart phone) \\
\hline 3 & Initially inexpensive & Initially expensive \\
\hline 4 & One-way communication & Interactive communication \\
\hline 5 & One-size-fits-all & Tailor target users \\
\hline 6 & General population & Market segmentation \\
\hline 7 & No IT skills needed & Basic skills needed \\
\hline 8 & No feedback & Allow feedback \\
\hline
\end{tabular}

\subsection{Low-Literacy Health Education}

Health literacy is the degree to which individuals have the capacity to obtain, process, and understand basic health information and services needed to make appropriate health decisions and follow instructions for treatment (American Medical Association Foundation, 2008). It is important to examine health literacy because low health literacy has been associated with poor health-related outcomes, which include hospitalization rates, poor adherence to prescribed treatment and self-care regimens, increase medication or treatment errors, failure to seek preventive care, lack of skills needed to navigate the health care system, disproportionately high rates of diseases and mortality, and the increased use of emergency rooms for primary care (H. L. Bankson, 2009; Choi \& Bakken, 2010).

A growing number of studies are now available to shed some light on the development and evaluation of practicable health care applications for low-literate users. For example, Ishibashi \& Nakajima (2004) developed a courseware to provide illiterate people with knowledge of preventing infectious diseases. They suggest that, rather than providing highly valuable satellite-assisted telemedicine systems to support high income doctors, a low valuable multimedia-assisted public health courseware will be far more cost-effective to engage in a project targeting poverty-stricken levels of society which are more susceptible to disease. It is undeniable that improving the knowledge of preventative measures of the low-literate population could reduce social risk and cost. Furthermore, Andersen et al. (2008) created an adaptive web-based kiosk system to deliver appropriate information about smoking cessation to lowliterate Hispanics (Mexican- Americans). The study was implemented in three stages, including a focus group interview, the development of a website, and a field testing and usability survey. The results showed that Hispanics were just as likely to use technology as Non-Hispanics, and that a kiosk was an appropriate way to collect data about the population of a clinic. In addition, Medhi et al. (2011) developed and tested three prototypes of mobile phone with a text-free interface, such as a spoken dialogue system, a graphical interface, and a live operator. They conducted an ethnographical study of the barriers to usability encountered by 90 low-literate subjects in India, Kenya, the Philippines, and South Africa. The results showed that first-time lowliterate users found textual interfaces to be unusable and the greatest number of tasks were completed using a graphical interface. They also found that a live operator was up to ten times more accurate than a text-based interface, and a spoken dialogue system could be used by those who felt more comfortable and familiar with speech. The 
broad range of the available testing methods makes it difficult to choose the best usability assessment plan, which is what the study and methodology described in this paper intends to investigate.

\subsection{Design for Low-Literate Users}

As the volume of health education materials rises, the need to evaluate its effectiveness becomes more critical. Evaluation is the crucial part of developing a healthcare application. It motivates the designer, and explains the guidelines related to concrete projects and problem-solving.

"Health message design is an audience-centred process; it is designed primarily to respond to the needs and situation of the target audience, rather than to the needs and situation of the message designers or sponsoring organizations." (Maibach \& Parrott, 1995, p167).

Nevertheless, how can the benefits of design be maximised to meet the special needs of poor readership? Scholars have produced a host of design guideline to help lowliterate patients to learn. These can be summarised as follows:

1. Information design: (1) Limit information to a specific objective; (2) Use short sentences and paragraphs; (4) Use plain language and avoid medical jargon; (3) Write in a conversational style; (5) Adopt a vivid and friendly tone; (6) Use headings and sub-headings to help reinforce the flow and content; (7) Provide an adequate amount of text on each page; (8) Divide the information content into several unique classifications.

2. Presentation design: (1) Ensure that the cover is attractive; (2) Use ample white spaces to clarify the layout; (3) Use a large font size to improve the readability of the content; (4) Closely link pictures to text or captions; (5) Minimise distracting details in pictures; (6) Use simple drawings to illustrate concepts; (7) Use real photos rather than fake cartoons; (8) Use bullets and other graphic devices to highlight key messages.

3. Interaction Design: (1) Interaction is invited via questions, responses, suggested action ; (2) Design user- friendly interfaces; (3) Provide users with control and freedom; (4) Consistency style of webpage design; (5)Voiding scrollbars, drop-down menus and multiple windows; (6) Use large link buttons adequately spaced apart; (7) Easy to recover from errors; (8) Short time to download and quickly feedback.

4. Appeal design: (1): The material closely matches the race, language, and experience of the target audience; (2) Offering information to meet users' needs; (3) The content of the website is easy to browse; (4) The source of the content is credible and up to date; (5) The authority and attribution are clearly recorded; (6)The advertisement and content are obviously compartmentalised; (7) Provide a discussion area for users to communicate with each other; (8) Offering professional on-line consultation.(Gillespie, 1993; Doak et al., 1996; Harvey\& Fleming, 2003; Houts et al., 2006; Andersen et al., 2008; Cassell et al., 2010; Green \& Tones, 2010; Choi \& Bakken, 2010; Hung \& Stones, 2011). 


\section{$3 \quad$ Methodology}

The study was implemented in five stages, including a focus group interview, the development of testing media, a reliability and validity test, a pre-post knowledge test, and a usability survey. Before commencing the primary research, ethics approval was granted by Fu Jen Catholic University and the University of Leeds.

Firstly, a focus group interview was conducted with 10 Vietnamese volunteer interpreters who have lived in Taiwan for years to provide a translation service for immigrant mothers consulting physicians. This ensured they had good knowledge of cultural and sociological issues the mothers experience on a regular basis. This brainstorming meeting at the Taipei Immigrant Hall was two hours in duration and explored the problems involved in health communication for low-literacy users and their media use. What was attained from their feedback served as a reference for the further development of usability testing media and the implementation of Pre-Post knowledge testing.

Secondly, a leaflet and a website were developed, both including the same healthcare information relating to the causation, symptoms, treatment and prevention of children's allergies. All the information and questionnaires for this research were created at secondary school level or lower to meet the standard of immigrant mothers' readability. All of the text, layout design and operating function in the testing media were integrated and converted using the computer software, Photoshop, Illustration, Flash and Dreamweaver, a combination of Adobe multimedia-development application systems.

Thirdly, a reliability and validity test was conducted by a paediatric allergy specialist, a paediatric clinical nurse, a Vietnamese translator, a new immigrant mother, and a professional researcher to ensure that all the questions in the questionnaire were good indicators to measure the important variables in this study. A 5-point Likert-type scale of response options ranging from strongly disagree to strongly agree was used to elicit endorsement of a particular statement, with higher scores indicating the higher appropriateness.

Fourthly, 64 immigrant mothers from Vietnam and China were randomly placed into two groups to explore the educational effectiveness of leaflets and websites respectively. The leaflet group was presented with the leaflet, whereas the website group was presented with the website. Both groups were asked to fill out the knowledge testing questionnaire before and after the trial. Thus, this research could measure their memory recall of the presented materials. The pre-test included demographic information and a questionnaire to test their knowledge. The post-test included the same questions as those contained in the pre-test, as well as a usability survey to assess the participants' user satisfaction.

Fifthly, each participant was asked to complete a questionnaire to evaluate the usability of the presented media. This consisted of 21 items of evaluation criteria, including the quality of information, presentation, and the appeal of the media in question. These evaluation criteria were informed by recent studies from evaluation literature and references collected from former focus group interviews. A five-point Likert Scale was used for every question, with higher scores indicating the higher 
appropriateness. The rating scale for measuring the appropriateness of the questionnaire was scored from $1=$ very unimportant, $2=$ unimportant, $3=$ no opinion, 4=important, 5=very important.

\subsection{Data Analysis}

1. In order to examine whether there were significant differences between the results shown in the pre-test and the post-test of using the leaflet as a media, a Pair T-test was applied. Table 1 shows the results: there were significant differences between the pre-test and the post-test of using the leaflet as a media. $(\mathrm{t}=-11.259$, *** $\mathrm{p}<.001)$

Table 1. Pair T-test (Leaflet)

\begin{tabular}{|l|l|l|l|l|}
\hline & & Mean & Mean Difference & t-value \\
\hline \multirow{2}{*}{ Leaflet } & Pre-test & 13.50 & 2.97 & $-11.259^{* * *}$ \\
\cline { 2 - 5 } & Post-test & 16.47 & & \\
\hline
\end{tabular}

2. In order to examine whether there were significant differences between the results shown in the pre-test and the post-test of using the website as a media, a Pair T-test was applied. Table 2 shows the results: there were significant differences between the pre-test and the post-test of using the website as a media. ( $\mathrm{t}=-9.063$, *** $\mathrm{p}<$ $.001)$

Table 2. Pair T-test (Website)

\begin{tabular}{|l|l|l|l|l|}
\hline & & Mean & Mean Difference & t-value \\
\hline \multirow{2}{*}{ Website } & Pre-test & 14.44 & \multirow{2}{*}{3.03} & $-9.063 * * *$ \\
\cline { 2 - 3 } & Post-test & 17.47 & & \\
\hline
\end{tabular}

3. In order to examine whether there were significant differences between the effectiveness of health intervention made by the leaflet and by the website, an independent sample t-test was applied. Table 3 shows the results: there was no significant difference between the effectiveness of health intervention made by the leaflet and by the website $(\mathrm{t}=-0.147, \mathrm{p}>.05)$.

Table 3. Independent Sample t-test (Leaflet VS. Website)

\begin{tabular}{|l|l|l|l|l|l|l|}
\hline & Media & $\mathrm{N}$ & Mean & Std. Deviation & Sig. & t-value \\
\hline $\begin{array}{l}\text { Educational } \\
\text { Effect }\end{array}$ & Leaflet & 32 & 2.97 & 1.49 & .884 & -1.147 \\
\cline { 2 - 6 } & Website & 32 & 3.03 & 1.89 & & - \\
\hline
\end{tabular}

4. In order to examine whether there were significant differences between the educational effect and the user's "educational level", One-Way ANOVA test was used. Table 4 shows the results: there were significant differences between the educational effect and the user's "educational level", $(\mathrm{F}=4.435, \mathrm{P}<.01)$. By using the Scheffe multiple-comparison test, we can see only those who acquired primary education and those who acquired secondary education show the significant differences. 
Table 4. One-Way ANOVA ( Educational Effect VS Education Level)

\begin{tabular}{|l|l|l|l|l|l|l|l|}
\hline & 1 & 2 & 3 & 4 & $\mathrm{~F}$ & Sig. & Scheffee Post Hoc Comparison \\
\hline Mean & 2.00 & 3.70 & 2.95 & 1.75 & 4.435 & $.007^{*}$ & $(1,2)^{*}$ \\
\hline
\end{tabular}

5. In order to examine the criteria used by low-literate patients to evaluate health educational material, Descriptive Statistics / Frequencies test was used. Chart 2 showed the results: Chart. 1. Usability Evaluation Survey
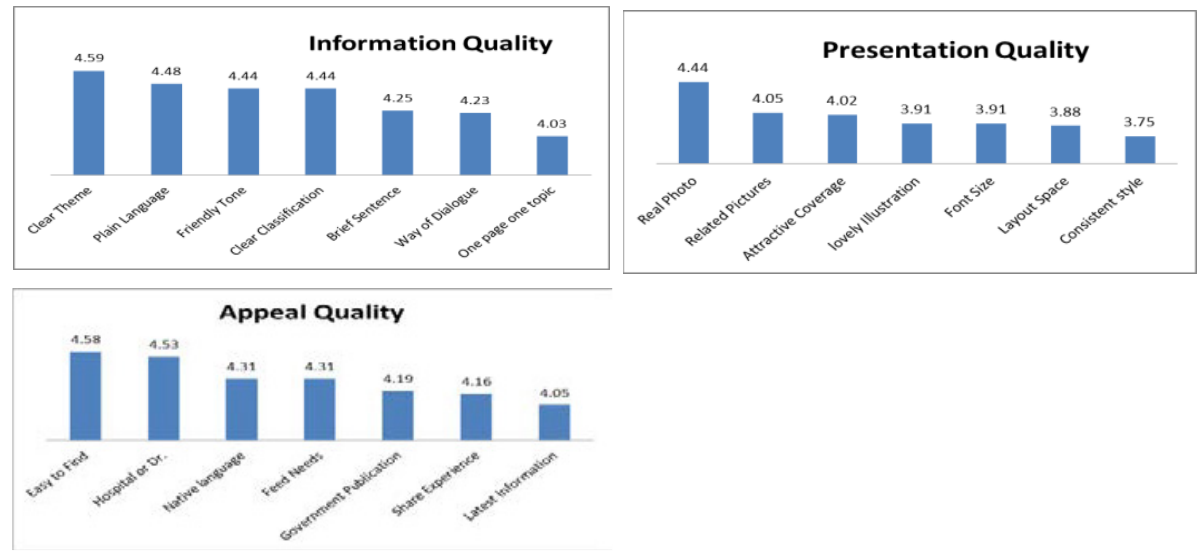

\section{Discussion and Conclusion}

The findings showed that the problems the immigrant mothers usually encountered in the clinic could be ranked as "which doctor to see", "cannot understand the prescription", "too shy to ask questions", "cannot understand what the doctor says", and "the doctor cannot understand what I say". This illustrates a fundamental concern to resolve the problems involved in doctor-patient communication among members of low-literate populations. The findings also showed that the most needed pediatric information for immigrant mothers when their children were sick can be ranked as "how to give care", "common symptoms", "trigger factors", "side effects", and "when to see a doctor". What is achieved from above might serve as a reference for further health promotional media design. Most of the immigrant mothers would ask their doctors, families and friends about the knowledge of children's healthcare, and only $48 \%$ of them indicated that they would make use of health educational materials. The media the immigrant mothers usually accessed to acquire information about their children's healthcare were ranked as television, brochures, books, internet, and DVD respectively. Moreover, most of the demographic factors did not have an impact on the educational effectiveness, apart from the user's "educational level". Low-literate users performed significantly better than highly literate ones, which proved that these two testing media had been well-designed for low-literate users and well-accepted by 
them. It is also probably because the users with high literacy are not as compliant as the users with low literacy to receive any suggestion from health educational materials. In addition, the results showed that the leaflet intervention had a positive outcome, as well as the website intervention. However, there was no significant difference between the influence of design intervention made by the leaflet and by the website. This is probably because both testing media were designed with the same content and were short of adequate probability sampling.

The findings also showed that the criteria used by low-literate patients to evaluate health educational material could be analyzed in terms of the quality of information, presentation, and appeal. Most of the participants agreed that the quality of information and appeal were more important than the quality of presentation. An assessment checklist of design of the health educational materials for low literacy patients is as follows:

1. Information design: 1 . It conveys clear themes; 2 . It is written in a friendly tone; 3. It uses plain language; 4. Information classification is very clear; 5. It represents information in the way of a dialogue; 6 . It has brief descriptive sentences; 7 . One topic is shown on one page

2. Presentation design: 1. Real photos help to understand information. 2. It has an attractive coverage (homepage); 3 . Related pictures are placed right next to the description; 4. The layout space of the content is well situated; 5 . The font size is suitable for reading; 6. Lovely illustrations are very attractive; 7. The colors and background are consistently designed.

3. Appeal design: 1 . If it is easy for users to find the information they want; 2 . If the media contains information of hospitals or doctors; 3 . If it is written in users' native language; 4. If it offers information to meet users' needs; 5. If someone else's caring experiences are related in the media; 6 . If it presents the latest information; 7. If it is published by the government.

This research has two major limitations, one of which is the use of probability sampling, while the other is the reliability of the questionnaire survey. It was very difficult to recruit low-literate participants because of the consent form for ethical reasons, while some feedback from the questionnaire may have been distorted because of embarrassing and upsetting issues. This research is an experiment that integrates design, communication and public health. Its findings are expected to be valuable, not only for providers and consumers, but also designers of health educational materials. Future studies are needed to identify which of the characteristics of low-literacy patients influence their ability to learn about health information. Identifying these factors and incorporating solutions into a health intervention may help to bridge the learning gap related to patients' literacy status.

Acknowledgement. The authors gratefully acknowledge the Taiwan National Science Council which funded this research, No: NSC 101-2410-H-030 -047 -SSS. 


\section{References}

1. Ahern, D., Kreslake, J., Phalen, J.: What is eHealth: Perspectives on the Evolution of eHealth Research. In: Krep, G.L. (ed.) Health Communication. Health Communication and new information technologies (eHealth), vol.4, pp. 267-287. SAGE Publications Ltd., London (2010)

2. American Medical Association Foundation. Health literacy. The Foundation, Boston (2008), http://www. ama-assn.org/ama/pub/category/8115.html (accessed: 150811 )

3. Andersen, P., Andersen, S., Youngblood, E., Colmenares, E.: Health education kiosk for low-literacy patients served by community-based clinics. In: 2008 IEEE International Symposium on Technology and Society, pp. 1-9 (2008)

4. Bankson, H.L.: Health Literacy: an exploratory bibliometric analysis, 1997-2007. J. Med. Libr. Assoc. 97(2) (April 2009)

5. Cassell, M., Jackson, C., Cheuvront, B.: Health Communication on the Internet: An Effective Channel for Health Behaviour Change? In: Krep, G.L. (ed.) Health Communication. Health Communication and new information technologies (eHealth), vol. 4, pp. 17-42. SAGE Publications Ltd., London (2010)

6. Choi, J., Bakken, S.: Web-based education for low-literate parents in Neonatal Intensive Care Unit: Development of a website and heuristic evaluation and usability testing. International Journal of Medical Informatics 79(8), 565-575 (2010)

7. Green, J., Tones, K.: Health Promotion: planning and strategies. Sage Publication (2010)

8. Houts, P., Doak, C., Doak, L., Loscalzo, M.: The role of pictures in improving health communication: A review of research on attention, comprehension, recall, and adherence. Patient Education and Counseling 61(2), 173-190 (2006)

9. Hung, Y.-L., Stones, C.: A Comparative Study of Children's eHealth Design between East and West: A Case Study of a Children's Health Website in China, Taiwan, the UK, and the US. In: Robertson, M.M. (ed.) EHAWC 2011 and HCII 2011. LNCS, vol. 6779, pp. 129138. Springer, Heidelberg (2011)

10. Ishibashi, Y., Nakajima, I.: The database which creates multilingual web information on preventing infectious diseases. In: Proceedings of the 6th International Workshop on Enterprise Networking and Computing in Healthcare Industry - Healthcom 2004 (IEEE Cat. No.04EX842), pp. 129-132 (2004)

11. Jan, R.-L., Wang, J.-Y., Huang, M.-C., Tseng, S.-M., Su, H.-J., Liu, L.-F.: An Internetbased interactive telemonitoring system for improving childhood asthma outcomes in Taiwan. Telemedicine Journal and e-Health 13(3), 257-268 (2007)

12. Kreuter, M., Strecher, V., Glassman, B.: One Size Does Not Fit All: The Case for Tailoring Print Materials. In: Krep, G.L. (ed.) Health Communication. Health Communication and health promotion, vol. 2, pp. 151-168. SAGE Publications Ltd., London (2010)

13. Maibach, E., Parrott, R.L. (eds.): Designing health messages: approaches from communication theory and public health practice, Thousand Oaks, Calif. Sage Publications, London (1995c)

14. Medhi, I., Patnaik, S., Brunskill, E., Gautama, S.N., Thies, W., Toyama, K.: Designing mobile interfaces for novice and low-literacy users. ACM Transactions on ComputerHuman Interaction 18(1) (April 2011)

15. Yardley, L., Morrison, L.G., Andreou, P., Joseph, J., Little, P.: Understanding reactions to an internet-delivered health-care intervention: accommodating user preferences for information provision. BMC Medical Informatics and Decision Making 10, 52 (2010) 